\title{
Skewness and Kurtosis of the Surface Wave in the Coastal Zone of the Black Sea
}

\author{
A. S. Zapevalov ${ }^{凶}$, A. V. Garmashov \\ Marine Hydrophysical Institute of RAS, Sevastopol, Russian Federation \\ 凶sevzepter@mail.ru
}

Purpose. The aim of the study is to analyze variability of the statistical moments characterizing deviations of the sea surface elevation distributions from the Gaussian.

Methods and Results. Field studies of the sea waves' characteristics were carried out from the stationary oceanographic platform located in the Black Sea near the Southern coast of Crimea. The data obtained both in summer and winter, were used. The statistical moments were calculated separately for wind waves and swell. The measurements were performed in a wide range of meteorological conditions and wave parameters (wind speed varied from 0 to $26 \mathrm{~m} / \mathrm{s}$, wave age from 0 to 5.2 and steepness - from 0.005 to 0.095). For wind waves, the coefficients of skewness correlation with the waves' steepness and age were equal to 0.46 and 0.38 . The kurtosis correlation coefficients with these parameters were small (0.09 and 0.07), but with the confidence level $99.8 \%$ significant. For swell, the correlation coefficients were $1.5-2.0$ times lower.

Conclusions. The statistical moments of the sea surface elevations of the third and higher orders are the indicators of the wave field nonlinearity, which should be taken into account when solving a wide range of the applied and fundamental problems. The deviations of the surface elevation distributions from the Gaussian one are not described unambiguously by the steepness and wave age. At the fixed values of these parameters, a large scatter in the skewness and kurtosis of the surface elevations is observed. This imposes significant limitations on the possibility of applying the nonlinear wave models based on the wave profile expansion by small parameter (steepness) degrees, in engineering calculations.

Keywords: sea surface, wind waves, swells, skewness, kurtosis, stationary oceanographic platform, Black Sea

Acknowledgments: the investigation was carried out within the framework of the state task on theme No. 0555-2021-0004 "Fundamental studies of the oceanological processes which determine the state and evolution of the marine environment influenced by natural and anthropogenic factors, based on the observation and modeling methods".

For citation: Zapevalov, A.S. and Garmashov, A.V., 2021. Skewness and Kurtosis of the Surface Wave in the Coastal Zone of the Black Sea. Physical Oceanography, [e-journal] 28(4), pp. 414-425. doi:10.22449/1573-160X-2021-4-414-425

DOI: 10.22449/1573-160X-2021-4-414-425

(C) A. S. Zapevalov, A. V. Garmashov, 2021

(C) Physical Oceanography, 2021

\section{Introduction}

It is customary to distinguish the two types of sea waves nonlinearity: statistical nonlinearity arising in the presence of bound wave components, and dynamic nonlinearity caused by resonant and quasi-resonant wave-wave interactions [1]. Both types of nonlinearity lead to the fact that the distribution of sea surface elevations deviates from the Gaussian distribution [2-4].

The nonlinearity of sea waves is weak, and the deviations of the sea surface elevations distribution from the Gaussian distribution are small [5, 6]. Despite their smallness, these deviations must be taken into account when calculating the impact 
of waves on vessels, oil and gas production platforms and coastal structures [7, 8], they play an important role in the occurrence of abnormally large waves $[9,10]$. Deviations from the Gaussian distribution must also be taken into account when analyzing the ocean remote sensing data [11, 12].

The third and higher cumulants of sea surface elevations can be used as parameters characterizing the nonlinearity of the wave field [13, 14]. Measurement of cumulants of the fifth order and higher in marine conditions is difficult, therefore, when constructing the probability density function of surface elevations, only cumulants of the third and fourth orders are used [15, 16]. Laboratory and field studies have shown that there are clear dependences of the sea surface elevations' cumulants on the steepness and the stage of the wave field development $[6,17,18]$. The existence of such dependences is confirmed by the modeling of nonlinear effects in the field of sea waves [4, 19].

The land and orography significantly affect wind fields, which, in its turn, directly affect the evolution of local wave fields [20-22]. For modeling and forecasting the wave climate in inland and marginal seas, testing the wave models, the information on the variability of local statistical characteristics of the surface waves are required [23].

The aim of this work is to analyze the variability of statistical parameters characterizing the deviations of the distributions of sea surface elevations from the Gaussian distribution in the Black Sea coastal zone.

\section{Instrumentation and measurement conditions}

The measurements were carried out on a stationary oceanographic platform located in the coastal part of the Black Sea off the Southern Coast of Crimea. The platform is a rectangular pile structure with horizontal dimensions of $20 \times 20 \mathrm{~m}$. The sea depth at the point where the platform is installed is about $\sim 30 \mathrm{~m}$. The upper deck, on which the laboratory rooms are situated, is located at a height of $12 \mathrm{~m}$, the height of the deck superstructures is $3 \mathrm{~m}$.

For the waveform measurements, a resistive wave recorder, the sensor of which is a nichrome string, wound with a constant pitch on a carrying strengthpower-communications cable. In order to minimize the disturbances of the wave field created by the platform supports, the wave recorder was positioned on a boom of $6 \mathrm{~m}$. Wind velocity measurements were carried out from a mast installed in the middle of the deck superstructure. The wind velocity and direction sensors were located at $21 \mathrm{~m}$ height. The description and characteristics of the measuring equipment are presented in [24]. The reduction of the wind velocity measured at $21 \mathrm{~m}$ height to 10 height accepted in meteorology was carried out according to the standard method under the assumption of a logarithmic profile [25].

The measurements were carried out in June and December 2018, as well as in January 2019. The wind velocity during the measurement period varied within $0-26 \mathrm{~m} / \mathrm{s}$, the age of the waves was $0-5.2$, and the steepness was $0.005-0.095$. For the analysis, the characteristics of the surface wave field averaged over $20 \mathrm{~min}$, as well as the characteristics of the wind velocity field, obtained simultaneously with the same averaging period, are used. The total data volume is more than 6500 measurement sessions. 


\section{Cumulants of the sea surface elevations}

We will consider a uniform stationary wave field in which the surface elevation at a fixed point is described by a random function with zero mean value $\eta=\eta(t)$, where $t$ is time. Distribution moments of order $n$ are described by the expression

$$
m_{n}=\int_{-\infty}^{\infty} \eta^{n} P(\eta) d x,
$$

where $P(\eta)$ - is a function of probability density functions. The first four cumulants are associated with the statistical moments by the relation

$$
\left.\begin{array}{l}
\lambda_{1}=m_{1}=0, \\
\lambda_{2}=m_{2}, \\
\lambda_{3}=m_{3}, \\
\lambda_{4}=m_{4}-3 m_{2}^{2} .
\end{array}\right\}
$$

For the Gaussian distribution

$$
\left.\begin{array}{c}
m_{2 n}=(2 n-1) ! ! m_{2}^{n}, \\
m_{2 n+1}=0, \quad n \geq 1,
\end{array}\right\}
$$

The equality to zero of cumulants senior than the second order makes them convenient for the analysis of nonlinear effects in the field of the sea surface waves $[13,14]$. When analyzing the data of field measurements, the asymmetry and kurtosis of the distribution of the sea surface elevations $\tilde{\lambda}_{3}=m_{3} / m_{2}^{3 / 2}$ and $\tilde{\lambda}_{4}=m_{4} / m_{2}^{2}-3$ are used.

We will use two dimensionless parameters as ones characterizing the evolution of the wave field. The first is the wave steepness

$$
\varepsilon=m_{2}^{1 / 2} k_{0},
$$

where $k_{0}$ is the wave number of the wave spectrum peak. In what follows, the waves with $k_{0}$ wavenumber will be called dominant. Dominant waves belong to the type of gravitational waves, for which the dispersion relation $\omega^{2}=g k$ is valid in deep water, whence it follows

$$
\varepsilon=m_{2}^{1 / 2} \omega_{0}^{2} / g,
$$

where $\omega_{0}$ is a frequency of dominant waves; $g$ is a gravitational acceleration. 
The deviation of the elevation distribution from the Gaussian distribution is also caused by the interaction of wind and waves. Obviously, the nature of the interaction will be different in the following stages: developing waves, fully developed waves, and swell. In what follows, we will use the parameter characterizing the age of the waves in the form

$$
\zeta=U_{10} / C_{0},
$$

where $U_{10}$ is a wind velocity at $10 \mathrm{~m}$ height; $C_{0}$ is a phase velocity of dominant waves [26, 27]. In the laboratory measurements a similar parameter $\zeta^{*}=u_{*} C_{0}$, where $u_{*}$ is a dynamic velocity [17], is used. The higher values of $\zeta$ (or $\zeta^{*}$ ) correspond to the earlier stages of the wind field development.

The location of the platform at a relatively small distance from the coast made it possible to measure the wave characteristics at the early stages of $\zeta>2$ wave field development, which is difficult to carry out in the open sea conditions. The early stages of development correspond to the situation when the wind blows from the coast. However, it should be taken into account that the structure of the near-water boundary layer changes with the wind from the coast, and turbulent eddies formed over the land have a significant effect on it [21].

We will consider two situations: when the wave field is formed by the wind waves and when it is formed by the swell. The swell is almost always observed on the sea surface, therefore this division is conditional. It is true insofar as the energy of the wind waves and swell differs. Completely developed field of surface waves corresponds to $\zeta_{0}=0.83$, at $\zeta>\zeta_{0}$ we will conventionally consider the waves to be the wind ones, at $\zeta<\zeta_{0}$ - the swell.
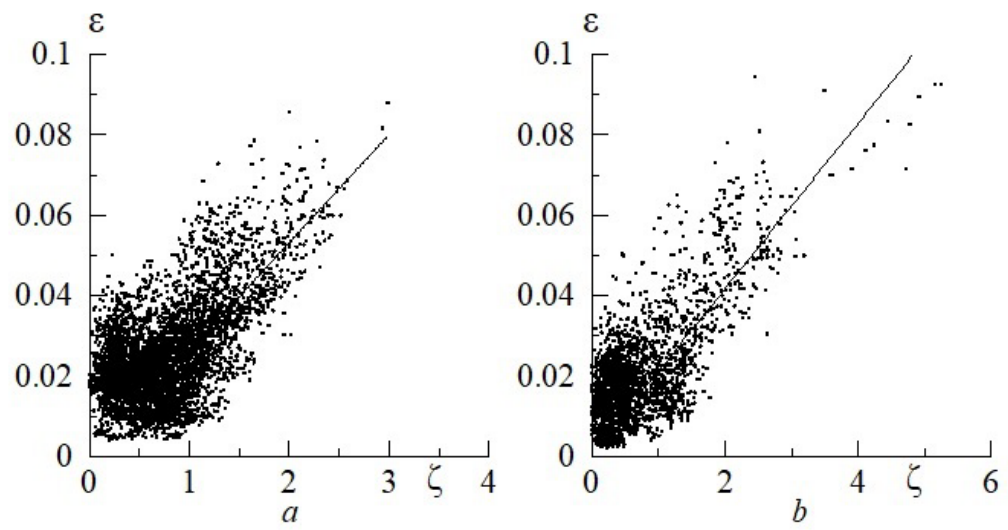

F i g. 1. Dependencies $\varepsilon=\varepsilon(\zeta)$ in the summer $(a)$ and winter $(b)$ periods

Variation of the wave steepness at different stages of the wave field development in winter and summer periods are presented in Fig. 1. Dependencies $\varepsilon=\varepsilon(\zeta)$ in the indicated periods are similar. The difference lies in the fact that in 
summer with the wind blowing from the coast, the range of $\zeta$ parameter variation is much wider than in winter.

For wind waves, the relationship between the parameters $\varepsilon$ and $\zeta$, plotted over the entire data set obtained in different periods, is approximated by the expression

$$
\varepsilon=0.021 \zeta
$$

The coefficient of correlation between $\varepsilon$ and $\zeta$ is equal to $r(\varepsilon, \zeta)=0.65$. For the swell

$$
\varepsilon=0.016+0.0061 \zeta
$$

and $r(\varepsilon, \zeta)=0.15$.

Different nature of the relationship between the parameters $\varepsilon$ and $\zeta$, which determine the deviations from the Gaussian distribution, indicates the need to analyze the variability of cumulants $\tilde{\lambda}_{3}$ and $\tilde{\lambda}_{4}$ separately for wind waves and swell.

\section{Skewness}

We begin the analysis of deviations from the Gaussian distribution by studying the dependence of skewness on the steepness and age of waves, these dependences are given in Fig. 2. The values of skewness mainly vary within the range of $-0.05 \ldots 0.3$. These limits are close to the boundaries of $\tilde{\lambda}_{3}$ variation within the range of $-0.05 \ldots 0.4$, recorded during the measurements carried out at the Ekofisk platform, located in the North Sea at $~ 70 \mathrm{~m}$ depth [16]. According to our measurements and measurements from the Ekofisk platform, the lower boundary of $\tilde{\lambda}_{3}$ variation lies in the negative area. This means that, in contrast to the classical representation of the sea wave (a pointed crest and a shallow depression), in some situations the reverse structure is observed. The values $\tilde{\lambda}_{3}<0$ were also obtained during the experiments in a research facility at low steepness [17].

The statistical characteristics of $\tilde{\lambda}_{3}$ relationship with the parameters describing the steepness and age of the waves, $\varepsilon$ and $\zeta$, are given in Table. 1, where $r$ is the correlation coefficient between $\tilde{\lambda}_{3}$ and the parameters $\varepsilon$ and $\zeta$; SD is a standard deviation. It also presents the coefficients of linear regressions $\tilde{\lambda}_{3}=a+b \varepsilon$ and $\tilde{\lambda}_{3}=a+b \zeta$ presented in Fig. 2. Statistical assessments lead to the following conclusions. The correlation coefficients between both $\tilde{\lambda}_{3}$ and parameters $\varepsilon$ and $\zeta$ for the wind waves are higher than for the swell waves. The steepness variations have a greater effect on the shape of the distribution of sea surface elevations than the wave age variations. 

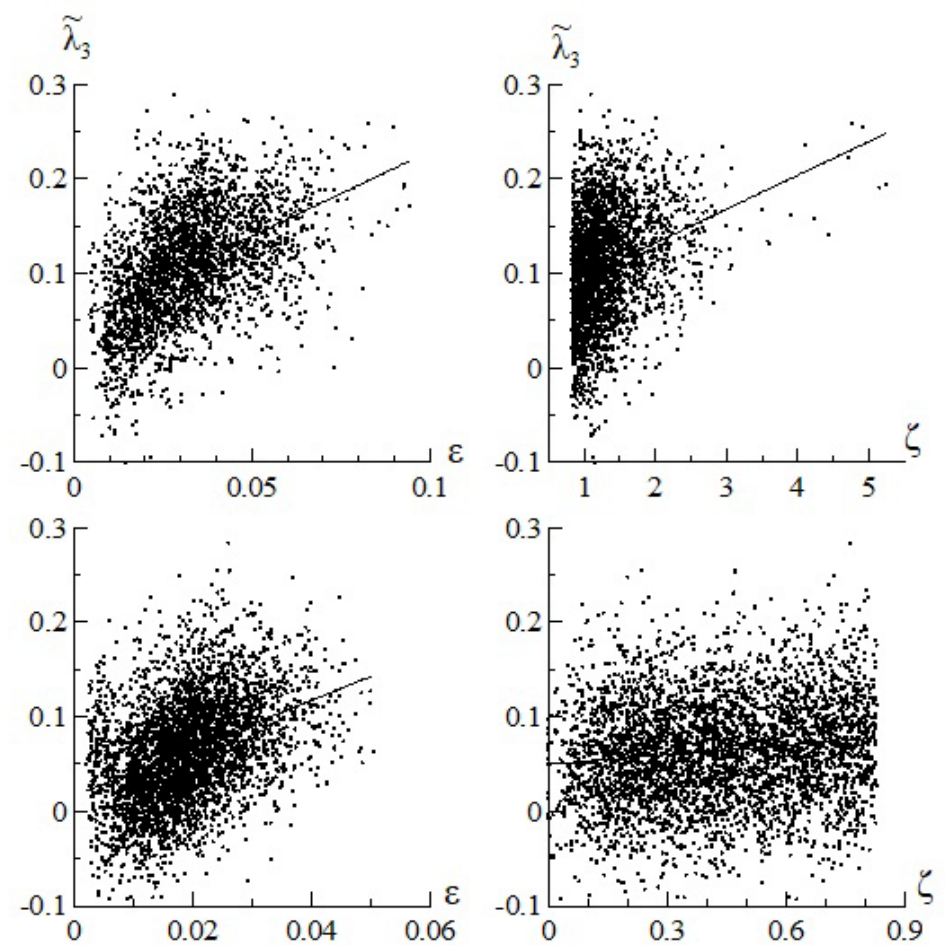

F i g. 2. Dependences of skewness $\tilde{\lambda}_{3}$ on the steepness $\varepsilon$ and wave age $\zeta$ (the upper row - wind waves, the lower one - swell; points are measurement data, lines are regressions)

T able 1

Statistical characteristics describing the skewness $\tilde{\lambda}_{3}$ dependences on the steepness $\varepsilon$ and wave age $\zeta$

\begin{tabular}{|c|c|c|c|c|}
\hline \multirow{2}{*}{ Parameters } & \multicolumn{3}{|c|}{ Coefficients } & \multirow{2}{*}{ SD } \\
\hline & $r$ & $a$ & $b$ & \\
\hline \multicolumn{5}{|c|}{ For wind waves } \\
\hline$\varepsilon$ & 0.46 & 0.050 & 1.795 & 0.052 \\
\hline$\zeta$ & 0.28 & 0.061 & 0.036 & 0.056 \\
\hline \multicolumn{5}{|c|}{ For swell } \\
\hline$\varepsilon$ & 0.38 & 0.016 & 2.547 & 0.051 \\
\hline$\zeta$ & 0.13 & 0.049 & 0.035 & 0.055 \\
\hline
\end{tabular}

\section{Kurtosis}

The kurtosis dependences on the steepness and stage of wave development are shown in Fig. 3. The kurtosis values for wind waves mainly vary within the range of - 0.4...0.4, which coincides with its variation limits obtained from PHYSICAL OCEANOGRAPHY VOL. 28 ISS. 4 (2021) 
the measurements on the Ekofisk platform [16]. Statistical characteristics describing the relationship of $\tilde{\lambda}_{4}$ and parameters $\varepsilon$ and $\zeta$ are given in Table 2. The structure and designations in Table 2 and Table 1 are similar.
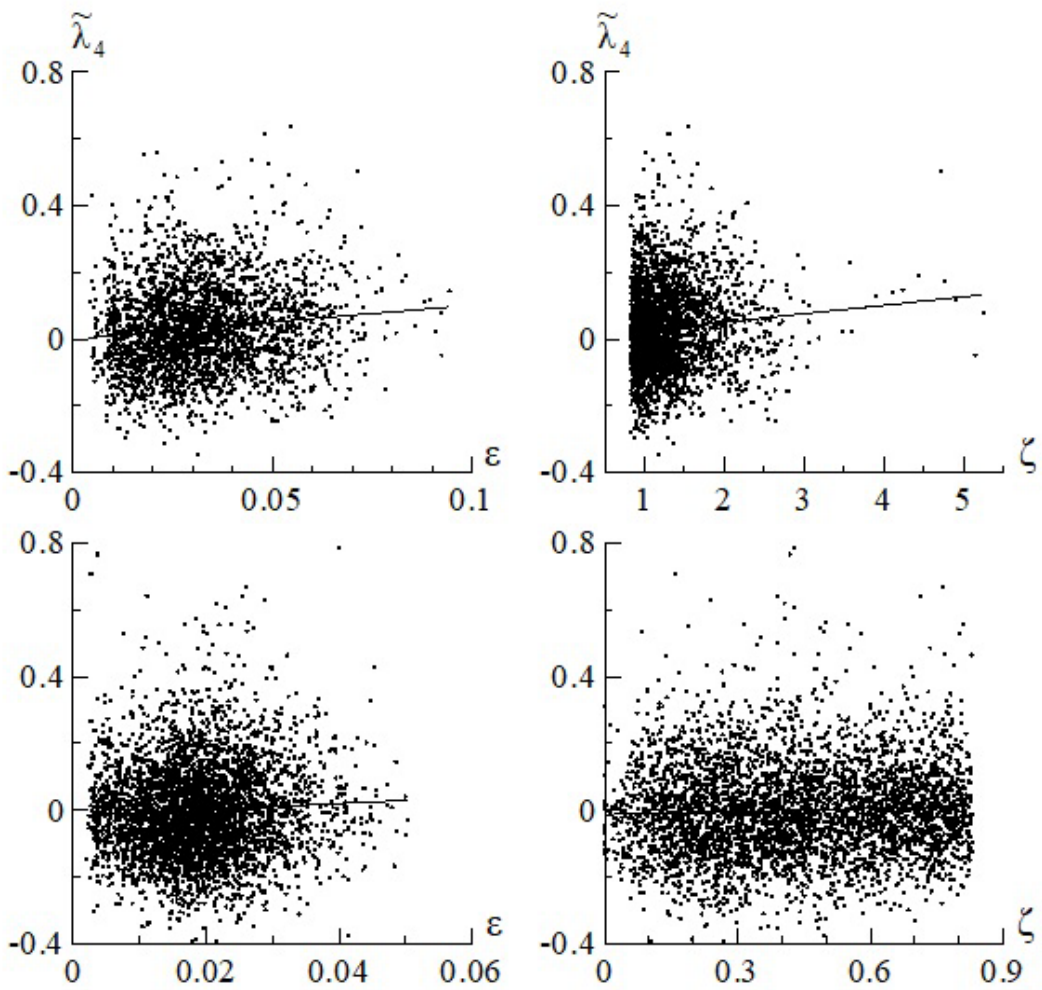

F i g. 3. Dependences of the kurtosis $\tilde{\lambda}_{4}$ on the steepness $\varepsilon$ and wave age $\zeta$ (the upper row - wind waves, the lower one - swell; points are measurement data, lines are regressions)

The coefficients of correlation between $\tilde{\lambda}_{4}$ and the parameters $\varepsilon$ and $\zeta$ are small, which determines the need to test the hypothesis that they are equal to zero. Evaluation based on the Student's test showed that for the wind waves with a probability of $99.8 \%$, the correlation coefficients $r\left(\tilde{\lambda}_{4}, \varepsilon\right)$ and $r\left(\tilde{\lambda}_{4}, \zeta\right)$ are significant. For swell waves, the correlation coefficient $r\left(\tilde{\lambda}_{4}, \varepsilon\right)$ is significant with a probability of $95 \%$, and the correlation coefficient $r\left(\tilde{\lambda}_{4}, \zeta\right)$ is significant with a probability of $90 \%$.

The studies of senior cumulants from the third to eighth order, carried out in the tray, showed that all cumulants depend on the steepness of the waves [17]. The exception was the fourth-order cumulant $\left(\tilde{\lambda}_{4}\right)$, for which no dependence was found. 
Statistical characteristics describing the kurtosis $\tilde{\lambda}_{4}$ dependences on the steepness $\varepsilon$ and wave age $\zeta$

\begin{tabular}{|c|c|c|c|c|}
\hline \multirow{2}{*}{ Parameters } & \multicolumn{3}{|c|}{ Coefficients } & \multirow{2}{*}{ SD } \\
\hline & $r$ & $a$ & $b$ & \\
\hline \multicolumn{5}{|c|}{ For wind waves } \\
\hline$\varepsilon$ & 0.09 & 0.006 & 0.865 & 0.14 \\
\hline$\zeta$ & 0.07 & 0.005 & 0.022 & 0.14 \\
\hline \multicolumn{5}{|c|}{ For swell } \\
\hline$\varepsilon$ & 0.05 & -0.016 & 0.881 & 0.15 \\
\hline$\zeta$ & 0.04 & -0.012 & 0.030 & 0.15 \\
\hline
\end{tabular}

\section{Discussion}

In recent years, in the analysis and forecasting of the sea surface waves, second-order nonlinear models based on the wave profile decomposition in a series in powers of a junior parameter $\varepsilon$ have become widespread $[8,14,16,19]$. In these models, elevation is represented as the sum of the linear and non-linear components. The linear component is a superposition of sinusoidal waves:

$$
\eta_{L}(x, t)=\sum_{n=1}^{\infty} a_{n} \cos \psi_{n}
$$

where $x$ is a spatial coordinate; $a_{n}$ is an amplitude; $\psi_{n}=k_{n} x-\omega_{n} t+\varphi_{n}, k_{n}$ and $\omega_{n}$ are wave number and angular frequency, $\varphi_{n}$ is a phase. Nonlinear component is described by the equation

$$
\eta_{N}(x, t)=\sum_{m=1}^{\infty} \sum_{n=1}^{\infty}\left\{a_{n} a_{n}\left[B_{m n}^{-} \cos \left(\psi_{m}-\psi_{n}\right)+B_{m n}^{+} \cos \left(\psi_{m}+\psi_{n}\right)\right]\right\},
$$

where $B_{m n}^{-}$and $B_{m n}^{+}$are the transfer functions of the second order, which are calculated from the Laplace equation for the velocity potential with nonlinear boundary conditions [16]. The nonlinear model takes into account the bound second-order components that appear as a result of the interaction of free waves.

The form of the probability density functions of the surface elevations constructed within the framework of the nonlinear model (1) depends only on one parameter - the steepness. Accordingly, the cumulants of the surface elevations are single-valued functions of the steepness, while from the measurement data (see Figs. 2 and 3) it follows that at the same $\varepsilon$ the values of $\tilde{\lambda}_{3}$ and $\tilde{\lambda}_{4}$ can differ significantly. Nonlinear models describe only the average trends in cumulant changes with a steepness variation, but do not allow describing the whole variety of situations that arise in marine conditions. Perhaps this is one of the reasons due to which these models have not become widespread in engineering calculations. 
As shown above, the statistical characteristics describing the relationship between the steepness and cumulants $\tilde{\lambda}_{3}$ and $\tilde{\lambda}_{4}$ are somewhat different for the wind waves and swell. We are to consider which situation is closer to the theoretical values of the cumulants obtained within the framework of the second-order nonlinear model. For comparison, we will use the analytical dependences given in [19]:

$$
\begin{aligned}
& \tilde{\lambda}_{3}{ }^{N}=3 \varepsilon+O\left(\varepsilon^{3}\right), \\
& \tilde{\lambda}_{4}{ }^{N}=12 \varepsilon^{2}+O\left(\varepsilon^{4}\right),
\end{aligned}
$$

where the superscript $N$ means that the cumulant is calculated using a nonlinear model. The results of comparison of theoretical and measured cumulants are demonstrated in Fig. 4. It can be seen that for the wind waves with increasing steepness, the values $\tilde{\lambda}_{3}{ }^{N}$ grow faster than $\tilde{\lambda}_{3}$. For $\varepsilon>0.08$, the values of $\tilde{\lambda}_{3}{ }^{N}$ go beyond the upper boundary of the region corresponding to the standard deviation from the regression. For the swell, the growth rates $\tilde{\lambda}_{3}{ }^{N}$ and $\tilde{\lambda}_{3}$ are approximately equal, the coefficients at $\varepsilon$ in equation (2) and in the linear regression equation differ by $20 \%$. The dependences $\tilde{\lambda}_{4}{ }^{N}=\tilde{\lambda}_{4}{ }^{N}(\varepsilon)$ and linear regressions $\tilde{\lambda}_{4}=\tilde{\lambda}_{4}(\varepsilon)$ for both wind waves and swell are close to each other.
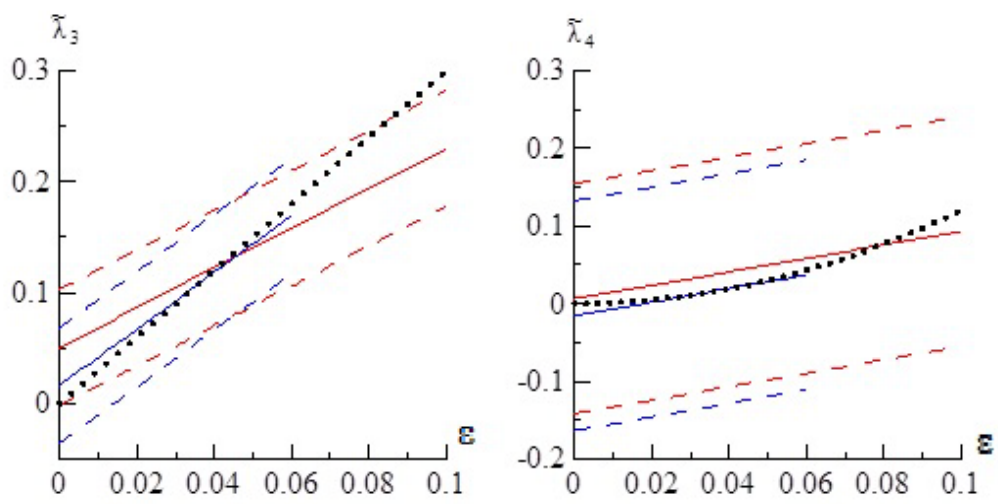

F i g. 4. Theoretical and measured dependences of the cumulants $\tilde{\lambda}_{3}$ and $\tilde{\lambda}_{4}$ on the waves' steepness $\varepsilon$ (red color shows the dependences obtained for wind waves, blue color - for swell; solid lines are the linear regressions, dashed ones denote STD; dotted line shows the dependencies (2) and (3), respectively)

\section{Conclusion}

Based on the data of direct wave measurements, an analysis of the deviations of the sea surface elevations' distributions from the Gaussian distribution is carried out. The measurements were carried out in summer and winter seasons in a wide range of variations in meteorological conditions and wave parameters (wind velocity varied within the range of $0-26 \mathrm{~m} / \mathrm{s}$, wave age $0-5.2$, and steepness 0.005-0.095). 
It was revealed that for the wind waves the correlation coefficients of the of the steepness kurtosis and the wave age kurtosis are equal to 0.46 and 0.38 . The kurtosis correlation coefficients with these parameters are small (0.09 and 0.07), but they are significant with a confidence level of 99.8\%. For the swell, the correlation coefficients are approximately one and a half to two times lower, $r\left(\tilde{\lambda}_{3}, \varepsilon\right)=0.38, r\left(\tilde{\lambda}_{3}, \zeta\right)=0.13, r\left(\tilde{\lambda}_{4}, \varepsilon\right)=0.05, r\left(\tilde{\lambda}_{4}, \zeta\right)=0.04$. The coefficients $r\left(\tilde{\lambda}_{4}, \varepsilon\right)$ and $r\left(\tilde{\lambda}_{4}, \zeta\right)$ are significant with a confidence level of 95 and $90 \%$, respectively.

It is concluded that the deviations of the surface elevations' distribution from the Gaussian distribution are not uniquely described by the steepness and age of the waves. At the fixed values of the steepness or the wave age, a large scatter in $\tilde{\lambda}_{3}$ and $\tilde{\lambda}_{4}$ values is observed. This imposes significant restrictions on the possibility of using nonlinear wave models (in particular, model (1)) based on the wave profile decomposition in powers of a small parameter (steepness) in engineering calculations.

The measurement data analyzed in this work were obtained in situations when the waves got at the measurement point both from the open sea and from the coast, i.e. under different wave formation conditions. The sea depth at the point where the measurements were taken corresponds to the "deep water" condition. This suggests that the conclusions drawn in this work will be true for other water areas as well.

\section{REFERENCES}

1. Taklo, T.M.A., Trulsen, K., Gramstad, O., Krogstad, H.E. and Jensen, A., 2015. Measurement of the Dispersion Relation for Random Surface Gravity Waves. Journal of Fluid Mechanics, 766, pp. 326-336. https://doi.org/10.1017/jfm.2015.25

2. Longuet-Higgins, M.S., 1963. The Effect of Non-Linearities on Statistical Distribution in the Theory of Sea Waves. Journal of Fluid Mechanics, 17(3), pp. 459-480. https://doi.org/10.1017/S0022112063001452

3. Phillips, O.M., 1961. On the Dynamics of Unsteady Gravity Waves of Finite Amplitude. Part 2. Local Properties of a Random Wave Field. Journal of Fluid Mechanics, 11(1), pp. 143-155. https://doi.org/10.1017/S0022112061000913

4. Socquet-Juglard, H., Dysthe, K., Trulsen, K., Krogstad, H.E. and Liu, J., 2005. Probability Distributions of Surface Gravity Waves during Spectral Changes. Journal of Fluid Mechanics, 542, pp. 195-216. https://doi.org/10.1017/S0022112005006312

5. Babanin, A.V. and Polnikov, V.G., 1995. On the Non-Gaussian Nature of Wind Waves. Physical Oceanography, 6(3), pp. 241-245. https://doi.org/10.1007/BF02197522

6. Zapevalov, A.S., Bol'shakov, A.N. and Smolov, V.E., 2011. Simulating of the Probability Density of Sea Surface Elevations Using the Gram-Charlier Series. Oceanology, 51(3), pp. 407-414. doi:10.1134/S0001437011030222

7. Song, J.-B., Wu, Y.-H. and Wiwatanapataphee, B., 2000. Probability Distribution of Random Wave Forces in Weakly Nonlinear Random Waves. Ocean Engineering, 27(12), pp. 13911405. doi:10.1016/s0029-8018(99)00067-0

8. Agarwal, P. and Manuel, L., 2009. On the Modeling of Nonlinear Waves for Prediction of Long-Term Offshore Wind Turbine Loads. Journal of Offshore Mechanics and Arctic Engineering, 131(4), 041601. doi:10.1115/1.3160647 
9. Xiao, W., Liu, Y., Wu, G. and Yue, D.K.P., 2013. Rogue Wave Occurrence and Dynamics by Direct Simulations of Nonlinear Wave-Field Evolution. Journal of Fluid Mechanics, 720, pp. 357-392. doi:10.1017/jfm.2013.37

10. Luxmoore, J., Ilic, S., and Mori, N., 2019. On Kurtosis and Extreme Waves in Crossing Directional Seas: A Laboratory Experiment. Journal of Fluid Mechanics, 876, pp. 792-817. doi:10.1017/jfm.2019.575

11. Gómez-Enri, J., Gommenginger, C.P., Challenor, P.G., Srokosz, M.A. and Drinkwater, M.R., 2006. ENVISAT Radar Altimeter Tracker Bias. Marine Geodesy, 29(1), pp. 19-38. https://doi.org/10.1080/01490410600582296

12. Zapevalov, A.S., 2012. Effect of Skewness and Kurtosis of Sea-Surface Elevations on the Accuracy of Altimetry Surface Level Measurements. Izvestiya, Atmospheric and Oceanic Physics, 48(2), pp. 200-206. https://doi.org/10.1134/S0001433812020120

13. Janssen, P.A.E.M., 2003. Nonlinear Four-Wave Interactions and Freak Waves. Journal of Physical Oceanography, 33(4), pp. 863-884. https://doi.org/10.1175/15200485(2003)33<863:NFIAFW>2.0.CO;2

14. Gao, Z., Sun, Z. and Liang, S., 2020. Probability Density Function for Wave Elevation Based on Gaussian Mixture Models. Ocean Engineering, 213, 107815. https://doi.org/10.1016/j.oceaneng.2020.107815

15. Zapevalov, A.S. and Ratner, Yu.B., 2003. Analytic Model of the Probability Density of Slopes of the Sea Surface. Physical Oceanography, 13(1), pp. 1-13. doi:10.1023/A:1022444703787

16. Jha, A.K. and Winterstein, S.R., 2000. Nonlinear Random Ocean Waves: Prediction and Comparison with Data. In: ASME, 2000. Proceedings of ETCE/OMAE2000 Joint Conference Energy for the New Millenium, February 14-17, 2000, New Orleans, LA, USA. New Orleans, USA, 2000. ETCE/OMAE 2000-6125. Available at: https://www.tamug.edu/sweetman/RMS_Papers/pdf/alok/6125.pdf [Accessed: 31 August 2021].

17. Huang, N. and Long, S., 1980. An Experimental Study of the Surface Elevation Probability Distribution and Statistics of Wind-Generated Waves. Journal of Fluid Mechanics, 101(1), pp. 179-200. doi:10.1017/S0022112080001590

18. Yijun, H., Guiting, S., Xixi, Z., Jinbao, S. and Quan'an, Z., 2006. Statistical Distribution of Nonlinear Random Water Wave Surface Elevation. Chinese Journal of Oceanology and Limnology, 24(1), pp. 1-5. doi:10.1007/BF02842767

19. Tayfun, M.A. and Alkhalidi, M.A., 2016. Distribution of Surface Elevations in Nonlinear Seas. In: OTC, 2016. Offshore Technology Conference Asia: proceedings. Kuala Lumpur, Malaysia. OTC-26436-MS. doi:10.4043/26436-MS

20. Efimov, V.V. and Komarovskaya, O.I., 2019. Disturbances in the Wind Speed Fields due to the Crimean Mountains. Physical Oceanography, 26(2), pp. 123-134. doi:10.22449/1573160X-2019-2-123-134

21. Solov'ev, Yu.P. and Ivanov, V.A., 2007. Preliminary Results of Measurements of Atmospheric Turbulence over the Sea. Physical Oceanography, 17(3), pp. 154-172. https://doi.org/10.1007/s11110-007-0013-9

22. Soloviev, Yu.P., 2013. Measurements of the Atmospheric Turbulence in the Coastal Zone of the Sea during Weak Wind from a Mountainous Coast. Izvestiya, Atmospheric and Oceanic Physics, 49(3), pp. 315-328. doi:10.1134/S0001433813030146

23. Cavaleri, L., Abdalla, S., Benetazzo, A., Bertotti, L., Bidlot, J.-R., Breivik, Ø., Carniel, S., Jensen, R.E. and Portilla-Yandun, J. [et al.], 2018. Wave Modelling in Coastal and Inner Seas. Progress in Oceanography, 167, pp. 164-233. doi:10.1016/j.pocean.2018.03.010

24. Toloknov, Yu.N., Korovushkin, A.I., 2010. [Hydrometeorological Information Collection System]. In: MHI, 2010. Monitoring Systems of Environment. Sevastopol: MHI. Iss. 13, pp. 50-53 (in Russian). 
25. Thomas, B.R., Kent, E.C. and Swail, V.R., 2005. Methods to Homogenize Wind Speeds from Ships and Buoys. International Journal of Climatology, 25(7), pp. 979-995. https://doi.org/10.1002/joc.1176

26. Donelan, M.A., Hamilton, J. and Hui, W.H., 1985. Directional Spectra of Wind-Generated Ocean Waves. Philosophical Transactions of the Royal Society of London. Series A, Mathematical and Physical Sciences, 315(1534), pp. 509-562. https://doi.org/10.1098/rsta.1985.0054

27. Young, I.R. and Donelan, M.A., 2018. On the Determination of Global Ocean Wind and Wave Climate from Satellite Observations. Remote Sensing of Environment, 215, pp. 228241. doi:10.1016/j.rse.2018.06.006

About the authors:

Aleksandr S. Zapevalov, Chief Research Associate, Marine Hydrophysical Institute of RAS (2 Kapitanskaya str., Sevastopol, 299011, Russian Federation), Dr. Sci. (Phys.-Math.), Scopus Author ID: 7004433476, WoS ResearcherID: V-7880-2017, sevzepter@mail.ru

Anton V. Garmashov, Senior Research Associate, Marine Hydrophysical Institute of RAS (2 Kapitanskaya str., Sevastopol, 299011, Russian Federation), Ph. D. (Geogr.), Scopus Author ID: 54924806400, WoS ResearcherID: P-4155-2017, ant.gar@mail.ru

Contribution of the co-authors:

Aleksandr S. Zapevalov - formulation of goals and objectives of the study; development of a statistical model; preparation of the paper text; editing and supplementing the paper text

Anton V. Garmashov - correcting the statistical model and carrying out the calculations; formalized and non-formalized data analysis; analysis and generalization of the research results

All the authors have read and approved the final manuscript.

The authors declare that they have no conflict of interest. 